\title{
Axillary Sampling as an Alternative Option for Complete Nodal Response in Triple Negative and HER2 Type Breast Cancer Patients after Neoadjuvant Chemotherapy
}

\author{
Chan Sub Park, M.D., Ph.D. ${ }^{1}$, Jeeyeon Lee, M.D., Ph.D. ${ }^{1}$, Jin Hyang Jung, M.D., Ph.D. ${ }^{1}$, \\ Wan Wook Kim, M.D., Ph.D. ${ }^{1}$, Yee Soo Chae, M.D., Ph.D. ${ }^{2}$, Hye Jung Kim, M.D., Ph.D. ${ }^{3}$, \\ Won Hwa Kim, M.D., Ph.D. ${ }^{3}$, Ji-Young Park, M.D., Ph.D. ${ }^{4}$, Jee Young Park, M.D., Ph.D. , \\ Soo Jung Lee, M.D., Ph.D. ${ }^{2}$, Mi Young Kim, M.D., Ph.D. ${ }^{5}$, Jung Dug Yang, M.D., Ph.D. ${ }^{6}$, \\ Joon Seok Lee, M.D., Ph.D. ${ }^{6}$, Ho Yong Park, M.D., Ph.D. ${ }^{1}$ \\ ${ }^{1}$ Department of Surgery, ${ }^{2}$ Department of Hemato-Oncology, ${ }^{3}$ Department of Radiology, ${ }^{4}$ Department of Pathology, ${ }^{5}$ Department of Radiation Oncology, \\ ${ }^{6}$ Department of Plastic and Reconstructive Surgery, School of Medicine, Kyungpook National University, Kyungpook National University Hospital, \\ Daegu, Korea
}

Purpose: In patients with locally advanced breast cancer, neoadjuvant chemotherapy is widely used. It has a distinct advantage in the downstaging of the primary tumor and provides important information about treatment response. With its increasing usage, concerns over the appropriate management of the axilla have emerged. In this study, we compared oncological outcomes of conventional axillary lymph node dissection (ALND) over axillary sampling (AS) with radiotherapy (RT) in patients who received neoadjuvant chemotherapy. Methods: In this retrospective study, we included female patients with triple negative breast cancer (TNBC) and HER2 type breast cancer who underwent breast and axillary surgery after neoadjuvant chemotherapy between May 2011 to December 2016. A total of 89 patients' medical records were eligible for analysis. We defined AS as removal of at least four axillary lymph nodes located near the sentinel lymph nodes without full exposure of the axillary vein, long thoracic nerve, and thoracodorsal nerve. Results: The median follow-up period was 47.00 months. The disease-free survival was 69.66 months in the AS with RT group and 69.02 months in the ALND group $(p=0.280)$. The invasive disease-free survival was 75.16 months in the AS with RT group and 78.44 months in the ALND group $(p=0.218)$. Conclusion: AS with radiotherapy might be a feasible surgical option in patients with TNBC and HER2 type breast cancer after neoadjuvant chemotherapy.

Key Words: Breast neoplasms, Lymph node excision, Neoadjuvant therapy

\section{INTRODUCTION}

In patients with locally advanced breast cancer, neoadjuvant chemotherapy is a common practice. Neoadjuvant chemotherapy has an advantage in primary tumor downstaging and provides important information about treatment response [1,2]. After neoadjuvant chemotherapy, approximately $40 \%-50 \%$ of patients with clinically $\mathrm{N} 1$ axillary node-positive disease are converted to clinically axillary node-negative disease [3-5]. Owing to recent advances in targeted therapy, neoadjuvant chemotherapy has resulted in an increased pathologically complete response. With the increased use of neoadjuvant chemotherapy

Correspondence: Ho Yong Park, M.D., Ph.D.

Department of Surgery, School of Medicine, Kyungpook National University, 807 Hoguk-ro, Buk-gu, Daegu 41404, Korea

Tel: +82-53-200-2702, Fax: +82-53-200-2028, E-mail: phy123@knu.ac.kr

Received: Apr 21, 2020 Revised: Aug 4, 2020 Accepted: Aug 22, 2020 and targeted therapy, concerns over appropriate management of the axilla have emerged [6-8] due to various rates of identification of the sentinel lymph node and the substantial false-negative rate of sentinel lymph node biopsy (5\%-30\%). However, interpretation of these studies is controversial as surgical techniques in most of these studies have not been well standardized [9-11].

Current studies, including The American College of Surgeons Oncology Group (ACOSOG) Z1071 trial, evaluate the accuracy of sentinel lymph node biopsy after neoadjuvant chemotherapy. In this trial, the false-negative rate for patients with $\mathrm{cN1}$ breast cancer after neoadjuvant chemotherapy and two or more sentinel lymph nodes examined was $12.6 \%[7,12,13]$. The European SENTinel neoadjuvant (SEN TINA) trial and the Canadian sentinel node biopsy following neoadjuvant chemotherapy (Sn FNAC) trial reported similar results $[8,14]$. To the best of our knowledge, few studies have compared the oncolog- 
ical outcomes between sentinel lymph node biopsy and axillary lymph node dissection (ALND) in patients treated with neoadjuvant chemotherapy [15]. While ALND remains the standard treatment for patients with clinically lymph node-positive disease after neoadjuvant chemotherapy, sentinel lymph node biopsy has been becoming a standard treatment for clinically lymph node-negative disease after neoadjuvant chemotherapy $[6-8,16,17]$. However, as proposed by researchers from the ACOSOG Z0011 trial, a less invasive axillary management strategy might be possible [3,18].

In patients with no residual disease after neoadjuvant chemotherapy, sentinel lymph node biopsy may not detect skip metastasis. Nonetheless, extensive axillary surgery might not provide better oncological benefit. However, the implementation of sentinel lymph node biopsy in patients treated with neoadjuvant chemotherapy remains a challenge. Recently, several researchers reported a new concept of axillary management, named axillary sampling (AS). AS includes limited or partial ALND; however, the concept and technique of AS are not yet clearly defined. Therefore, the clinical usefulness of AS is limited [19-22]. Nodal irradiation after sentinel lymph node biopsy has been proven as a safe alternative to ALND among patients with one or two metastatic lymph nodes undergoing surgery [23]. Rates of nodal pCR with neoadjuvant chemotherapy differ based on tumor subtype, ranging from $40 \%$ to $60 \%$ overall, and approaching $70 \%$ to $80 \%$ among patients with triple negative and HER2 type tumors [24-26]. Therefore, AS with radiotherapy (RT) could be safe for patients with triple negative and HER2 type breast cancer.

The aim of this study was to compare the oncological outcomes between conventional ALND and AS with radiotherapy in triple negative breast cancer (TNBC) and HER2 type breast cancer patients treated with neoadjuvant chemotherapy.

\section{METHODS}

\section{Definition of ALND and AS}

Conventional ALND was defined as the gross removal of most of the axillary lymph nodes with exposure of the axillary vein, long thoracic nerve, and thoracodorsal nerve. For identification of the sentinel lymph node, we used radiolabeled colloid, blue dye, or a combination of these methods. A gamma probe identified radioactivity in the lymph nodes in the axilla, and blue-stained lymphatic nodes were vi- sually identified by surgeons. Additionally, the axilla was meticulously examined and any palpable or visually abnormal lymph nodes were resected and submitted for frozen biopsy. Clinically, AS was defined as the removal of several axillary lymph nodes located near the sentinel lymph nodes without full exposure of the axillary vein, long thoracic nerve, and thoracodorsal nerve. Additionally, based on ACOSOG Z1071 and many other clinical trials, we defined that AS should include removal of at least four lymph nodes [7,8,12-14].

\section{Patients and surgery}

In this retrospective study, we selected subjects from among 2,332 patients who had breast and axillary surgery between May 2011 to December 2016. Stage IV and clinical trial patients was excluded. Patients who received neoadjuvant chemotherapy were selected and among these, TNBC and HER2 type breast cancer patients were selected. Total of 89 patients' medical records were eligible for analysis. These data included immunohistochemical analyses for hormonal receptor status, HER2 status, and other clinical factors, including age at the time of diagnosis, tumor size, lymph node status, and patient outcomes. After chemotherapy, axillary nodal status was re-evaluated with breast sonography and magnetic resonance imaging. This study was approved by the Institutional Review Board (IRB No. KNUCH 2019-06-012). Informed consent was waived due to the retrospective nature of the study.

\section{Diagnosis and treatment}

All cases of breast cancer were diagnosed by needle or excisional biopsy and axillary lymph node metastasis was confirmed by fine-needle aspiration cytology. The size, number, and location of the tumor were identified through mammography, ultrasonography, and breast magnetic resonance imaging prior to surgery. All patients provided written informed consent to undergo neoadjuvant chemotherapy, breast surgery, and axillary surgery. Conventional ALND included the removal of level I and II axillary lymph nodes. During AS, patients received ALND when more than four metastatic lymph nodes were detected with frozen biopsy. For ALND group, most of cases were planned ALND. Adjuvant radiotherapy was recommended to patients who received AS, and patients with $\mathrm{pN} 2$ after ALND were recommended to undergo adjuvant radiotherapy. 


\section{Surveillance}

After surgery, all patients were followed up with radiological imaging and intensive physical examination biannually for the first two years and annually for another three years. Tumor recurrence or me- tastasis was evaluated with blood tests, tumor markers, mammography, breast sonography, breast magnetic resonance imaging, chest X-ray, and bone scan.

Table 1. Clinicopathologic characteristics of patients $(n=89)$

\begin{tabular}{|c|c|c|c|c|}
\hline Characteristic & & $\begin{array}{l}\text { Axillary sampling } \\
\text { with radiotherapy } \\
\qquad \begin{array}{l}(n=25) \\
\text { No. }(\%)\end{array}\end{array}$ & $\begin{array}{c}\text { Axillary } \\
\text { dissection } \\
(n=64) \\
\text { No. }(\%)\end{array}$ & $p$-value \\
\hline Age $(y r)^{*}$ & & $49.16 \pm 9.56$ & $49.19 \pm 8.93$ & 0.925 \\
\hline \multirow[t]{4}{*}{ Clinical T stage } & 1 & $2(8.0)$ & $7(10.9)$ & 0.817 \\
\hline & 2 & $16(64.0)$ & $37(57.8)$ & \\
\hline & 3 & $5(20.0)$ & $17(26.6)$ & \\
\hline & 4 & $2(8.0)$ & $3(4.7)$ & \\
\hline \multirow[t]{4}{*}{ Clinical N stage } & 0 & $3(12.0)$ & $1(1.6)$ & 0.161 \\
\hline & 1 & $9(36.0)$ & $21(32.8)$ & \\
\hline & 2 & $9(36.0)$ & $26(40.6)$ & \\
\hline & 3 & $4(16.0)$ & $16(25.0)$ & \\
\hline \multirow[t]{2}{*}{ Clinical N stage after chemotherapy } & 0 & $19(76.0)$ & $42(65.6)$ & 0.343 \\
\hline & 1 & $6(24.0)$ & $22(34.4)$ & \\
\hline \multirow[t]{5}{*}{ Pathologic T stage } & 0 & $7(28.0)$ & $18(28.1)$ & 0.990 \\
\hline & is & $3(12.0)$ & $8(12.5)$ & \\
\hline & 1 & $9(36.0)$ & $20(31.3)$ & \\
\hline & 2 & $4(16.0)$ & $13(20.3)$ & \\
\hline & 3 & $2(8.0)$ & $5(7.8)$ & \\
\hline \multirow[t]{2}{*}{ Pathologic N stage } & 0 & $18(72.0)$ & $39(60.9)$ & 0.462 \\
\hline & 1 & $7(28.0)$ & $25(39.1)$ & \\
\hline Harvested nodes* & & $6.48 \pm 2.83$ & $15.38 \pm 7.26$ & $<0.001$ \\
\hline \multirow{4}{*}{ Histologic grade } & 1 & 0 & $1(1.6)$ & 0.877 \\
\hline & 2 & $17(68.0)$ & $39(60.9)$ & \\
\hline & 3 & $7(28.0)$ & $21(32.8)$ & \\
\hline & Unknown & $1(4.0)$ & $3(4.7)$ & \\
\hline Triple negative breast cancer & & $7(28.0)$ & $24(37.5)$ & 0.465 \\
\hline HER2 type & & $18(72.0)$ & $40(62.5)$ & 0.465 \\
\hline \multirow[t]{5}{*}{ Chemotherapy regimen } & AC & $1(4.0)$ & 0 & 0.024 \\
\hline & AC followerd by taxol & $7(28.0)$ & $31(48.4)$ & \\
\hline & AC followerd by taxol with trastuzumab & $12(48.0)$ & $30(46.9)$ & \\
\hline & Docetaxel+Doxorubicin & 0 & $1(1.6)$ & \\
\hline & Docetaxel+Carboplatin+Trastuzumab+Pertuzumab & $5(20.0)$ & $2(3.1)$ & \\
\hline \multirow[t]{2}{*}{ Breast surgery } & Breast conserving surgery & $7(28.0)$ & $16(25.0)$ & 0.792 \\
\hline & Mastectomy & $18(72.0)$ & $48(75.0)$ & \\
\hline \multirow[t]{2}{*}{ Adjuvant radiotherapy } & Yes & $22(88.0)$ & $49(76.6)$ & 0.378 \\
\hline & No & $3(12.0)$ & $15(23.4)$ & \\
\hline \multirow[t]{3}{*}{ Axillary radiotherapy } & Yes & $17(68.0)$ & $9(14.1)$ & $<0.001$ \\
\hline & No & $5(20.0)$ & $40(62.5)$ & \\
\hline & Unknown & $3(12.0)$ & $15(23.4)$ & \\
\hline \multirow[t]{4}{*}{ Types of recurrence } & Local & 0 & $1(1.6)$ & 0.403 \\
\hline & Regional & $2(8.0)$ & $3(4.7)$ & \\
\hline & Distant & $2(8.0)$ & $14(21.9)$ & \\
\hline & Contralateral breast & 0 & $2(3.1)$ & \\
\hline Death & & $2(8.0)$ & $5(7.8)$ & 0.976 \\
\hline
\end{tabular}

HER2 = human epidermal growth factor receptor 2; AC = adriamycin+cyclophosphamide.

*Mean \pm SD. 


\section{Statistical analysis}

The clinical variables assessed included age, type of breast cancer, clinical and pathological tumor size, number of metastatic and removed axillary lymph nodes, overall cancer stage, and results of immunohistochemical staining. Oncological results included diseasefree survival, locoregional recurrence, distant metastasis, and death.

For comparison of ALND and AS with RT, Pearson's chi-square test or Student t-test were used, depending on the variables. Diseasefree survival was defined as the time from diagnosis to the first event ending disease-free survival, including locoregional recurrence, distant metastasis, contralateral breast cancer, other primary cancer, or death from any other cause. Invasive disease-free survival was defined as the time from diagnosis to the first event showed distant metastasis or death from any other cause. Survival analysis was constructed using the Kaplan-Meier method and differences were assessed using the logrank test. Cox regression analysis was used in the univariate and multivariate analyses. Statistical significance was accepted for a $p$-value of $<0.05$.

\section{RESULTS}

\section{Patient characteristics}

The median follow-up period was 47.00 months (range: 9-96 months). Clinicopathologic characteristics of the patients are listed in Table 1. A total of 25 patients received AS with RT (28.1\%). In both groups, clinical T, N stage, pathologic T, N stage showed no significant difference. Post neoadjuvant chemotherapy clinical $\mathrm{N}$ stage ( $\mathrm{ycN}$

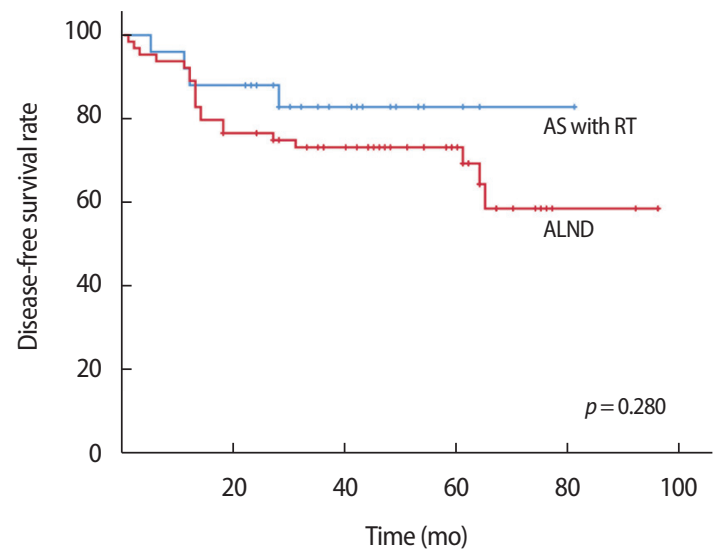

Figure 1. Kaplan-Meier plots for disease-free survival comparing the axillary sampling (AS) with radiotherapy (RT) group and the axillary lymph node dissection (ALND) group. stage) showed no significant difference. Pathological characteristics showed no significant difference between the two groups. Neoadjuvant chemotherapy regimen showed difference between both groups. In AS with RT group, $68.0 \%$ of patients received axillary radiotherapy whereas, $14.1 \%$ of patients received axillary radiotherapy in ALND group.

\section{Survival analysis according to types of axillary surgery}

The disease-free survival was 69.66 months in the AS with RT group and 69.02 months in the ALND group. There were no statistically significant differences between the two groups $(p=0.280$ ) (Figure 1). The invasive disease-free survival was 75.16 months in the AS with RT

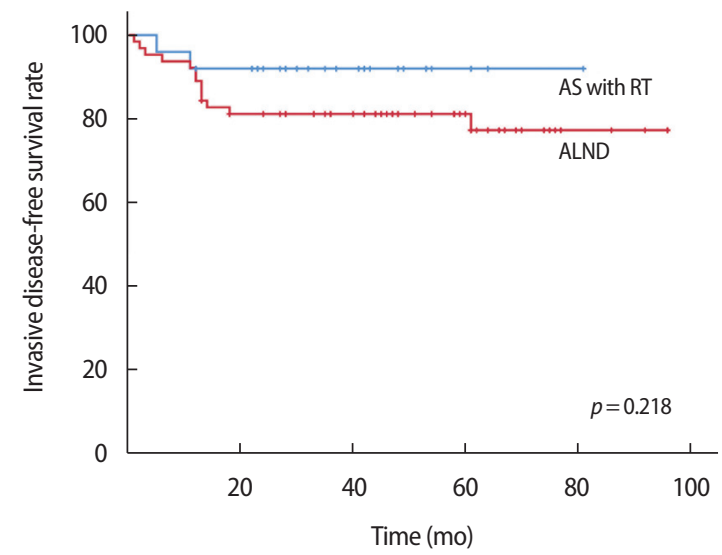

Figure 2. Kaplan-Meier plots for invasive disease-free survival comparing the axillary sampling (AS) with radiotherapy (RT) group and the axillary lymph node dissection (ALND) group.

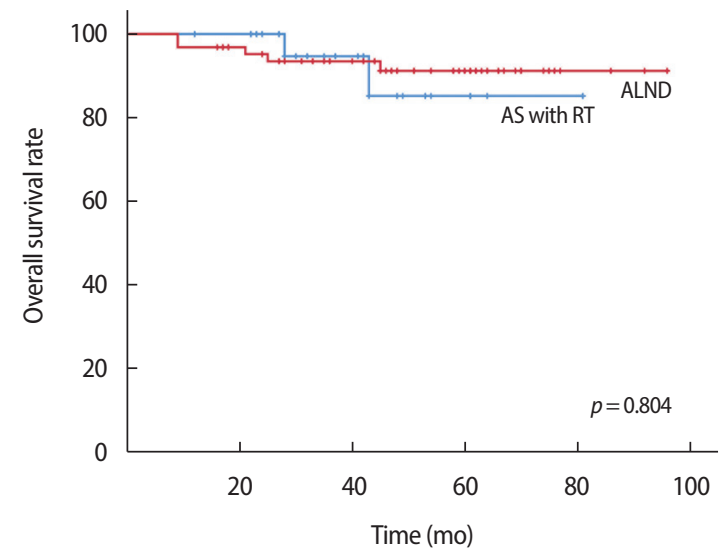

Figure 3. Kaplan-Meier plots for overall survival comparing the axillary sampling (AS) with radiotherapy (RT) group and the axillary lymph node dissection (ALND) group. 
Table 2. Univariate and multivariate analysis of associated factors in invasive disease-free survival

\begin{tabular}{|c|c|c|c|c|c|c|}
\hline \multirow{2}{*}{ Factor } & \multicolumn{3}{|c|}{ Univariate analysis } & \multicolumn{3}{|c|}{ Multivariate analysis } \\
\hline & Hazard ratio & $95 \% \mathrm{Cl}$ & $p$-value & Hazard ratio & $95 \% \mathrm{Cl}$ & $p$-value \\
\hline AS+RT vs. ALND & 2.465 & $0.555-10.956$ & 0.236 & & & \\
\hline cN 0,1 vs. 2,3 & 4.277 & 0.963-18.989 & 0.056 & 4.685 & $1.053-20.837$ & 0.043 \\
\hline ycN 0 vs. others & 2.040 & $0.739-5.631$ & 0.169 & & & \\
\hline ypN 0 vs. others & 4.173 & $1.414-12.315$ & 0.010 & 4.476 & $1.516-13.211$ & 0.007 \\
\hline Ki67 $\leq 14$ vs. $>14$ & 1.160 & $0.319-4.215$ & 0.822 & & & \\
\hline
\end{tabular}

$\mathrm{Cl}=$ confidence interval; $\mathrm{AS}=$ axillary sampling; $\mathrm{RT}=$ radiotherapy; $\mathrm{ALND}=$ axillary lymph node dissection; $\mathrm{CN}=$ clinical $\mathrm{N}$ stage; $\mathrm{ycN}=$ post neoadjuvant chemotherapy clinical $\mathrm{N}$ stage; $\mathrm{ypN}=$ post neoadjuvant chemotherapy pathologic $\mathrm{N}$ stage.

group and 78.44 months in the ALND group. There were no statistically significant differences between the two groups $(p=0.218$ ) (Figure 2). The overall survival was 74.61 months in the AS with RT group and 89.68 months in the ALND group. There was no statistically significant difference between the two groups $(p=0.804)$ (Figure 3).

\section{Univariate and multivariate analysis for associated factors}

We investigated the related factor for invasive disease-free survival. In univariate analysis, post neoadjuvant chemotherapy pathologic $\mathrm{N}$ stage (ypN 0 vs. others, hazard ratio 4.173, $p=0.010$ ) is correlated with invasive disease-free survival. AS with RT vs. ALND exhibited no significant relationship with invasive disease free survival. In multivariate analysis, clinical $\mathrm{N}$ stage ( $\mathrm{cN} \mathrm{0,1} \mathrm{vs.} \mathrm{2,3,} \mathrm{hazard} \mathrm{ratio} \mathrm{4.685,} p=$ 0.043 ) and post neoadjuvant chemotherapy pathologic $\mathrm{N}$ stage (ypN 0 vs. others, hazard ratio $4.476, p=0.007$ ) had strong prognostic significance for invasive disease free survival (Table 2).

\section{DISCUSSION}

Axillary lymph node dissection is an important element in the surgical management of breast cancer because it provides staging information and removes metastatic lymph nodes. However, ALND is associated with lymphedema, arm pain, sensory defects, decreased range of motion, and other morbidities that could impair quality of life. Sentinel lymph node biopsy can decrease the risk of these morbidities and has become a standard method for the evaluation of axillary lymph nodes [19-22,27]. However, ALND remains standard care for patients with a clinically positive lymph node before neoadjuvant chemotherapy.

Recently, it has been questioned whether ALND should be performed in breast cancer cases with only one to three metastatic lymph nodes. The American College of Surgeons Oncology Group (ACOSOG) Z0011 trial, the International Breast Cancer Study Group (IBCSG) 23-01 trial, and the Comparison of Complete Axillary Lymph node Dissection With Axillary Radiation Therapy in Treating Women With Invasive Breast Cancer (AMAROS) trial provided suggestions about the management of axillary lymph nodes $[18,23,28,29]$. Since then, many surgeons have considered less invasive techniques than ALND, including AS, sentinel lymph node dissection, or partial ALND [19-22]. Although AS could reduce the complications associated with ALND, many surgeons do not accept the concept due to lack of evidence for its oncological safety and it's unclear definition. These issues may impact current comparison studies of AS.

We defined AS in clinical and pathological ways through a multidisciplinary team discussion. AS included dissection of the sentinel lymph nodes and other lymph nodes in proximity to the sentinel lymph nodes. In this study, we attempted to remove more than two lymph nodes, including the sentinel lymph nodes and other surrounding nodes. According to some prospective trials (ACOSOG Z1071 trial, SENTINA trial, Sn FNAC trial), removing more than two lymph nodes could reduce the false-negative rate $[7,8,14]$. In our results, the mean number of lymph nodes removed was $6.48 \pm 2.83$ in the AS with RT group. Using this method, the accuracy of axillary lymph node staging may be sufficiently obtained. Axillary RT field included axillary lymph node level I, II, and III. Internal mammary lymph node and supraclavicular lymph node could be included in the treatment field. This RT field definition was following ESTRO consensus guidelines [30].

The aim of this study was to compare the oncological outcomes between AS with RT and ALND with TNBC in HER2 type breast cancer after neoadjuvant chemotherapy. Our results show that disease- 
free survival, invasive disease-free survival, and overall survival were not significantly different between the AS with RT group and the ALND group. Based on these results, AS with RT could satisfy oncological safety in patients with a clinically complete axillary nodal response after neoadjuvant chemotherapy.

In our study result, the axillary complete nodal response was $64.0 \%$ for all patients and was consistent with other studies [3-5]. This study indicates that at least $50 \%$ of patients who received neoadjuvant chemotherapy may not need ALND. The morbidity of axillary dissection is a great concern for both patients and clinicians; thus, the selection of appropriate patients is important. Several nomograms were developed for this purpose; $[15,31]$ however, these nomograms require further external validation and sentinel lymph node detection methods warrant greater standardization.

Some of the strengths of our study include a reduction in the falsenegative rates associated with AS due to sentinel lymph node biopsy only. In addition, we utilized frozen biopsy samples to detect metastatic lymph nodes. Patients with more than three metastatic lymph nodes underwent ALND, reducing the possibility of under treatment.

The utilization of frozen biopsy samples to detect metastatic lymph nodes allowed us to select patients who did not need ALND. Our retrospective study has a limitation owing to the study design. However, results from this study provide evidence to support future prospective randomized controlled trials evaluating the role of AS with RT in patients who received neoadjuvant chemotherapy. Another limitation of this study was the ambiguous definition of AS. Despite our attempt at defining AS in clinical and pathological terms, a clear definition of the procedure has yet to be established.

AS with radiotherapy might be a feasible surgical option for patients with TNBC and HER2 type breast cancer after neoadjuvant chemotherapy. In addition, AS may be an alternative modality to reduce multiple morbidities caused by ALND. However, as this surgical technique should be applied carefully, the selection of appropriate patients is crucial.

\section{CONFLICT OF INTEREST}

The authors declare that they have no competing interests.

\section{ACKNOWLEDGMENTS}

This work was supported by the National Research Foundation of Korea (NRF) grant funded by the Korea government (2014R1A5A2009242, 2017R1C1B5076186) and by a grant from the National R\&D Program for Cancer Control, Ministry of Health and Welfare, Republic of Korea (1420040). In addition, this research was supported by a grant of the Korea Health Technology R\&D Project through the Korea Health Industry Development Institute (KHIDI), funded by the Ministry of Health \& Welfare, Republic of Korea (HI17C1142). This work was also supported by the National Research Foundation of Korea (NRF) grant funded by the Korea government (MSIT) (NRF2019R1A2C1006264), as well as the National Research Foundation of Korea (NRF) grants funded by the Korea government (2017M3A9G8083382), and the Foundation of Korea (NRF) grant funded by the Korea government (2019R1F1A1063853).

\section{REFERENCES}

1. Mauri D, Pavlidis N, Ioannidis JP. Neoadjuvant versus adjuvant systemic treatment in breast cancer: a meta-analysis. J Natl Cancer Inst 2005;97:188-94

2. Jones RL, Smith IE. Neoadjuvant treatment for early-stage breast cancer: opportunities to assess tumour response. Lancet Oncol 2006; 7:869-74

3. Giuliano AE, Morrow M, Duggal S, Julian TB. Should ACOSOG Z0011 change practice with respect to axillary lymph node dissection for a positive sentinel lymph node biopsy in breast cancer? Clin Exp Metastasis 2012;29:687-92.

4. Vila J, Mittendorf EA, Farante G, Bassett RL, Veronesi P, Galimberti $\mathrm{V}$, et al. Nomograms for predicting axillary response to neoadjuvant chemotherapy in clinically node-positive patients with breast cancer. Ann Surg Oncol 2016;23:3501-9.

5. Bilimoria KY, Bentrem DJ, Hansen NM, Bethke KP, Rademaker AW, Ko CY, et al. Comparison of sentinel lymph node biopsy alone and completion axillary lymph node dissection for node-positive breast cancer. J Clin Oncol 2009;27:2946-53.

6. Mamounas EP, Brown A, Anderson S, Smith R, Julian T, Miller B, et al. Sentinel node biopsy after neoadjuvant chemotherapy in breast cancer: results from national surgical adjuvant breast and bowel 
project protocol B-27. J Clin Oncol 2005;23:2694-702.

7. Boughey JC. Sentinel lymph node surgery after neoadjuvant chemotherapy in patients with node-positive breast cancer. JAMA 2013; 310.

8. Kuehn T, Bauerfeind I, Fehm T, Fleige B, Hausschild M, Helms G, et al. Sentinel-lymph-node biopsy in patients with breast cancer before and after neoadjuvant chemotherapy (SENTINA): a prospective, multicentre cohort study. Lancet Oncol 2013;14:609-18.

9. Newman EA, Sabel MS, Nees AV, Schott A, Diehl KM, Cimmino VM, et al. Sentinel lymph node biopsy performed after neoadjuvant chemotherapy is accurate in patients with documented node-positive breast cancer at presentation. Ann Surg Oncol 2007;14:2946.

10. Classe JM, Bordes V, Campion L, Mignotte H, Dravet F, Leveque J, et al. Sentinel lymph node biopsy after neoadjuvant chemotherapy for advanced breast cancer: results of ganglion sentinelle et chimiothérapie neoadjuvante, a French prospective multicentric study. J Clin Oncol 2009;27:726-32.

11. Alvarado R, Yi M, Le-Petross H, Gilcrease M, Mittendorf EA, Bedrosian I, et al. The role for sentinel lymph node dissection after neoadjuvant chemotherapy in patients who present with node-positive breast cancer. Ann Surg Oncol 2012;19:3177-84.

12. Boughey JC, Ballman KV, Hunt KK, McCall LM, Mittendorf EA, Ahrendt GM, et al. Axillary ultrasound after neoadjuvant chemotherapy and its impact on sentinel lymph node surgery: results from the American college of surgeons oncology group Z1071 trial (alliance). J Clin Oncol 2015;33:3386-93.

13. Boughey JC, Ballman KV, Le-Petross HT, McCall LM, Mittendorf EA, Ahrendt GM, et al. Identification and resection of clipped node decreases the false-negative rate of sentinel lymph node surgery in patients presenting with node-positive breast cancer (T0-T4, N1-N2) who receive neoadjuvant chemotherapy: results from ACOSOG Z1071 (alliance). Ann Surg 2016;263:802-7.

14. Boileau JF, Poirier B, Basik M, Holloway CM, Gaboury L, Sideris L, et al. Sentinel node biopsy after neoadjuvant chemotherapy in biopsy-proven node-positive breast cancer: the SN FNAC study. J Clin Oncol 2015;33:258-64.

15. Ryu JM, Lee SK, Kim JY, Yu J, Kim SW, Lee JE, et al. Predictive factors for nonsentinel lymph node metastasis in patients with positive sentinel lymph nodes after neoadjuvant chemotherapy: nomogram for predicting nonsentinel lymph node metastasis. Clin Breast Can- cer 2017;17:550-8

16. Barnwell JM, Arredondo MA, Kollmorgen D, Gibbs JF, Lamonica D, Carson W, et al. Sentinel node biopsy in breast cancer. Ann Surg Oncol 1998;5:126-30.

17. Brenin DR, Morrow M, Moughan J, Owen JB, Wilson JF, Winchester DP. Management of axillary lymph nodes in breast cancer: a national patterns of care study of 17,151 patients. Ann Surg 1999;230:686.

18. Giuliano AE, Hunt KK, Ballman KV, Beitsch PD, Whitworth PW, Blumencranz PW, et al. Axillary dissection vs no axillary dissection in women with invasive breast cancer and sentinel node metastasis: a randomized clinical trial. JAMA 2011;305:569-75.

19. Salmon RJ, Marcolet A, Vieira M, Languille O. Sentinel node biopsy or limited oriented axillary dissection (LOAD) in early breast cancer. Eur J Surg Oncol 2005;31:949-53.

20. Lumachi F, Norberto L, Zanella S, Marino F, Basso SMM, Basso U, et al. Axillary node sampling in conjunction with sentinel node biopsy in patients with breast cancer. A prospective preliminary study. Anticancer Res 2011;31:693-7.

21. Bassi KK, Seenu V, Srivastava A, Al Sharara N. Role of axillary sampling in the era of sentinel lymph node biopsy: a critical review. Indian J Cancer 2012;49:66-73.

22. Li J, Jia S, Zhang W, Qiu F, Zhang Y, Gu X, et al. Partial axillary lymph node dissection inferior to the intercostobrachial nerves complements sentinel node biopsy in patients with clinically node-negative breast cancer. BMC Surg 2015;15:79.

23. Donker M, van Tienhoven G, Straver ME, Meijnen P, van de Velde CJH, Mansel RE, et al. Radiotherapy or surgery of the axilla after a positive sentinel node in breast cancer (EORTC 10981-22023 AMAROS): a randomised, multicentre, open-label, phase 3 non-inferiority trial. Lancet Oncol 2014;15:1303-10.

24. Boughey JC, McCall LM, Ballman KV, Mittendorf EA, Ahrendt GM, Wilke LG, et al. Tumor biology correlates with rates of breastconserving surgery and pathologic complete response after neoadjuvant chemotherapy for breast cancer: findings from the ACOSOG Z1071 (alliance) prospective multicenter clinical trial. Ann Surg 2014;260:608-14.

25. Kim JY, Park HS, Kim S, Ryu J, Park S, Kim SI. Prognostic nomogram for prediction of axillary pathologic complete response after neoadjuvant chemotherapy in cytologically proven node-positive breast cancer. Medicine 2015;94:e1720. 
26. Diego EJ, McAuliffe PF, Soran A, McGuire KP, Johnson RR, Bonaventura M, et al. Axillary staging after neoadjuvant chemotherapy for breast cancer: a pilot study combining sentinel lymph node biopsy with radioactive seed localization of pre-treatment positive axillary lymph nodes. Ann Surg Oncol 2016;23:1549-53.

27. Lee J, Jung JH, Kim WW, Lee RK, Kim HJ, Kim WH, et al. 5-year oncological outcomes of targeted axillary sampling in pT1-2N1 breast cancer. Asian J Surg 2019;42:681-7.

28. Morrow M, Giuliano AE. To cut is to cure: can we really apply Z11 in practice? Ann Surg Oncol 2011;18:2413-5.

29. Galimberti V, Cole BF, Zurrida S, Viale G, Luini A, Veronesi P, et al.
Axillary dissection versus no axillary dissection in patients with sentinel-node micrometastases (IBCSG 23-01): a phase 3 randomised controlled trial. Lancet Oncol 2013;14:297-305.

30. Offersen BV, Boersma LJ, Kirkove C, Hol S, Aznar MC, Biete Sola A, et al. ESTRO consensus guideline on target volume delineation for elective radiation therapy of early stage breast cancer. Radiother Oncol 2015;114:3-10.

31. Jeruss JS, Newman LA, Ayers GD, Cristofanilli M, Broglio KR, Meric-Bernstam F, et al. Factors predicting additional disease in the axilla in patients with positive sentinel lymph nodes after neoadjuvant chemotherapy. Cancer 2008;112:2646-54. 